\title{
Generation of Human Bronchial Epithelial Cell Lines Expressing Inactive Mutants of GALNT3
}

\author{
Shoko NAKAMURA ${ }^{1,2)}$, Masayuki HORIE ${ }^{1), * *}$, Kan FUJINO $^{1)}$, Yusuke MATSUMOTO ${ }^{1)}$, Tomoyuki HONDA ${ }^{1)}$ and \\ Keizo TOMONAGA ${ }^{1), *}$ \\ 1) Department of Viral Oncology, Institute for Virus Research, Kyoto University, Kyoto 606-8507, Japan \\ 2) Department of Biomedical Informatics, Division of Health Sciences, Osaka University Graduate School of Medicine, Suita, Osaka \\ 565-0871, Japan
}

(Received 2 May 2012/Accepted 29 May 2012/Published online in J-STAGE 12 June 2012)

ABSTRACT. As a tool to understand the role of mucins in the infection of respiratory viruses, we established cell lines stably expressing inactive mutants of UDP-GalNAc:polypeptide N-acetylgalactosaminyltransferase 3 (GALNT3), which initiates O-glycosylation of mucins. We introduced single amino acid mutations into the regions essential for the enzyme activity of GALNT3 using the expression plasmid of human GALNT3 and transfected the mutant constructs into a human bronchial epithelial cell line, BEAS-2B. We showed that although the mutants of GALNT3 exhibit an authentic localization at the Golgi apparatus, the glycosylation pattern of the expressing cell lines appeared to be different from that of the cells expressing wild-type GALNT3. These results suggested that the established cell lines express inactive forms of GALNT3 and might be useful in investigation of the significance of O-glycosylation of mucins in respiratory virus infections.

KEY WORDS: GALNT3, mucin, respiratory viruses.

doi: 10.1292/jvms.12-0199; J. Vet. Med. Sci. 74(11): 1493-1496, 2012

Viral infections affecting the upper or lower respiratory tract, such as influenza virus, parainfluenza virus, adenovirus and respiratory syncytial virus, commonly induce characteristic alterations in the epithelial surfaces of the respiratory tract $[2,6,13]$. One such change is the increased production of mucus [14]. The increased mucus in the respiratory tract is believed to be involved in many characteristic clinical symptoms, as well as viral eliminations, at the early stage of virus infections [8]. The mucus is largely formed by mucins, which are complex O-linked glycosylated proteins. Recent studies revealed that the expression of mucins increases in the epithelial cells both in vivo and in vitro soon after some respiratory virus infections [9] and also that the upregulation of mucins may play important roles for the host defense mechanisms against virus infections [12]. However, the details about how mucins are involved in the host defense mechanisms have not yet been elucidated.

The O-glycosylation of mucins is initiated by the transfer of GalNAc to serine and threonine (Ser/Thr) residues on target polypeptides by a family of UDP-GalNAc:polypeptide $\mathrm{N}$-acetylgalactosaminyltransferase (GalNAc-T or GALNT). In humans, the GALNT family consists of twenty genes, which localize to different chromosomal loci. The genes

\footnotetext{
*Correspondence to: Tomonaga, K., Department of Viral Oncology, Institute for Virus Research, Kyoto University, Sakyo-ku, Kyoto 606-8507, Japan. e-mail: tomonaga@virus.kyoto-u.ac.jp

**PRESENT ADDRESS: HoRIE M., Department of Virology, Institute for Medical Microbiology and Hygiene, University of Freiburg, D-79104 Freiburg, Germany.

(C)2012 The Japanese Society of Veterinary Science
}

have different but overlapping substrates and expression specificities [16]. Among them, GALNT3 is considered to mediate $\mathrm{O}$-glycosylation of some mucins, including MUC1 and MUC2, as well as fibroblast growth factor 23 (FGF23), which is a bone-derived hormone that plays an important role in regulation of the metabolism of phosphate and 1,25-dihydroxy vitamin D $[1,7,15]$. Previous studies demonstrated that the expression of GALNT3 is seen in several normal tissues, including respiratory epithelium cells and bronchial glands cells [3], showing suggestive roles of GALNT3 in respiratory virus infections. In this study, to understand the roles of mucin-type O-glycosylation in the infection of respiratory viruses, we tried to generate human bronchial epithelial cell lines expressing inactive mutants of GALNT3.

To generate an expression plasmid of human GALNT3, cDNA corresponding to the GLANT3 coding region was amplified from total RNA isolated from human alveolar basal epithelial cells, A549, by PCR and cloned into the restriction enzyme sites (Kpn I and Not I) of the pEF4 plasmid to yield pEF-hGALNT3. Detailed information about the primers and PCR procedures used to generate the plasmid is available from the authors. The nucleotide sequence of the recombinant plasmid was confirmed by DNA sequencing. To confirm the expression of recombinant GALNT3, the plasmid was transfected into a human bronchial epithelial cell line, BEAS-2B and detected by anti-GALNT3 polyclonal antibody (Sigma-Aldrich St. Louis, MO, U.S.A.) (Fig. 2B, see below). The inactive mutants of GALNT3 were constructed by introducing single amino acid substitutions using pEF-hGALNT3. To predict the reactive residues within GALNT3, we used information from earlier studies $[4,16,17]$, in which the amino acid residues responsible 


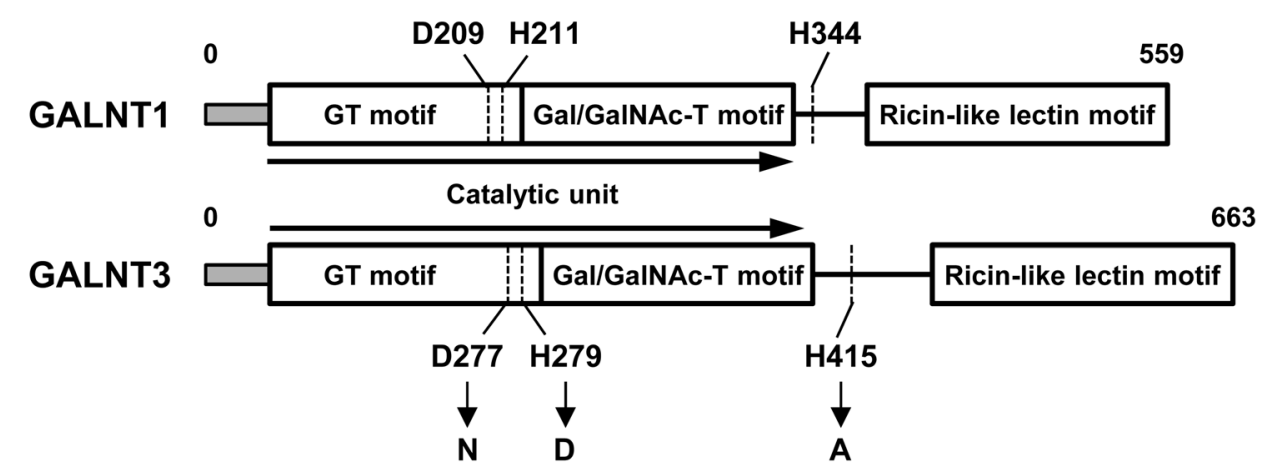

Fig. 1. Schematic representation of GALNT1 and GALNT3. The regions of the N-terminal catalytic unit, which contain GT and Gal/GalNAc-T motifs, and the C-terminal ricin-like lectin motif are shown. The residues essential for the enzyme activity of GALNT1 (murine and bovine) and their corresponding sites in GALNT3 (human) are indicated. The amino acids used for the substitution in each site in GALNT3 are indicated by arrows.

for the enzyme activity of murine and bovine GalNAc-T1 (GALNT1) were determined. As the GALNT family is known to be highly homologous and the amino acid residues essential for the enzyme activity seem to be significantly conserved among the family [16], we introduced mutations in human GALNT3 in the sites corresponding to those identified in murine and bovine GALNT1 (Fig. 1). In previous studies, some residues (mostly histidines) were identified as essential for the enzyme activity of GALNT1 [4]. Among them, mutants of aspartate and histidine at positions 209 and 211 of murine GALNT1, respectively, consist of a DxH motif, which was also described in many other glycosyltransferase, and showed no detectable enzyme activity [17]. In addition, a point mutation of histidine at position 344 was shown to significantly decrease the activity of bovine GALNT1 [17]. Therefore, we introduced the point mutations in the corresponding residues, D277, H279 and H415, of human GALNT3 (Fig. 1). According to the previous studies, the aspartate and histidine residues were changed to asparagine (D277N), aspartate (H279D) and alanine (H415A), respectively. The mutant forms of the expression plasmid were generated using PCR amplification and recloning or a PCRbased site-directed mutagenesis technique. Oligonucleotide primers and PCR conditions to create the plasmids are available on request. Nucleotide sequences of the recombinant constructs were also confirmed by DNA sequencing.

To generate cell clones stably expressing the GALNT3 mutants, we first tested the susceptibility of BEAS-2B cells to respiratory virus infection. The A/Puerto Rico/8/34 (PR8) strain of influenza A virus was inoculated into the parental BEAS-2B cell line at a multiplicity of infection (MOI) of 3.0, and infectivity was confirmed by indirect immunofluorescence assay (IFA) using anti-nucleoprotein (NP) monoclonal antibody. As shown in Fig. 2A, at $12 \mathrm{hr}$ postinfection, the parental BEAS-2B cells were shown to be susceptible to influenza $A$ virus. In addition, endogenous expression of GALNT3 was undetectable in the parental BEAS-2B cells by both Western blot analysis and IFA (Fig. 2B and 2C), indicating that BEAS-2B cells are relevant for studying the role of GALNT3 in respiratory virus infection.

The mutant plasmids were transfected into BEAS-2B cells, and cell clones stably expressing the wild-type and mutant GALNT3 were selected using $200 \mu \mathrm{g} / \mathrm{m} l$ Zeocin (Invitrogen, Carlsbad, CA, U.S.A.) in D-MEM (Dulbecco's Modified Eagle's Medium) (Nacalai Tesque, Kyoto, Japan). We obtained 4, 3 and 7 cell clones expressing D277N, H279D and H415A mutants, respectively, in which the expression levels, as well as the intracellular localization, of the mutant proteins were comparable to those of the wild-type GALNT3 (data not shown). Among them, we used a representative clone in each mutant, and the expression of the mutant enzymes was verified by Western blotting and IFA using anti-GALNT3 polyclonal antibody (Sigma-Aldrich). Immunoblotting using $12 \%$ SDS-PAGE revealed that the mutants produce recombinant proteins at the expected sizes (Fig. 2B). Previous studies revealed that the glycosyltransferases commonly localize at the Golgi apparatus [11]. As shown in Fig. 2C, all the mutants, D277N, H279D and H415A, as well as the wild-type protein, exhibited a clear distribution in the Golgi apparatus. These results indicated that the single amino acid substitutions exert no effects on the expression and localization of GALNT3 in the transfected cells.

We next investigated whether the GALNT3 mutants showed reduced enzyme activity in the cells. To this end, we performed lectin blot analysis using lysates of BEAS-2B cell lines stably expressing the wild-type and mutant GALNT3. Briefly, the cells were washed with PBS and then lysed. Insoluble materials were removed by centrifugation, and equal amounts of protein were separated using $12 \%$ SDS-PAGE and then transferred to nitrocellulose membranes (Millipore). As a control for protein loading, we performed Coomassie Brilliant Blue (CBB) staining using CBB Stain One (Nacalai Tesque) (Fig. 3). For lectin blotting, membranes were blocked with a Carbo-Free Blocking Solution (Vector Laboratories, Inc. Burlingame, CA, U.S.A.), and incubated with a different biotinylated lectin, $1.0 \mathrm{mg} / \mathrm{m} l$ PNA (Arachis hypogaea agglutinin) (Sigma-Aldrich) or $1.0 \mathrm{mg} / \mathrm{m} l \mathrm{ABA}$ (Agaricus bisporus agglutinin) (Vector Laboratories, Inc.) in 
A

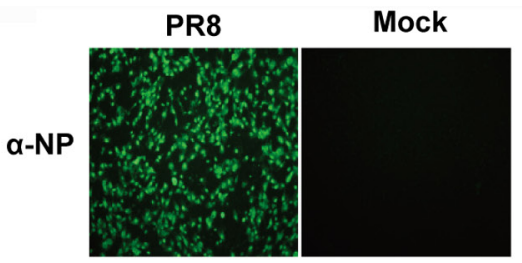

B
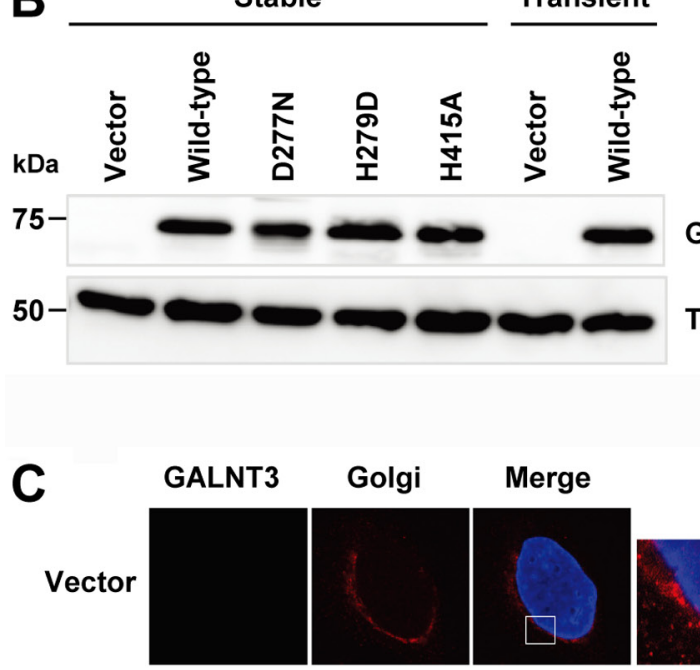

Merge
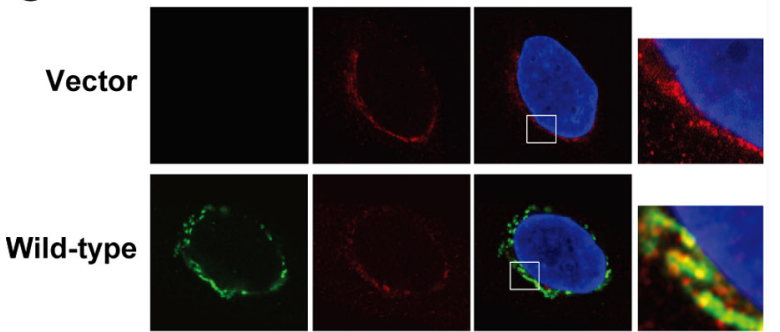

D277N
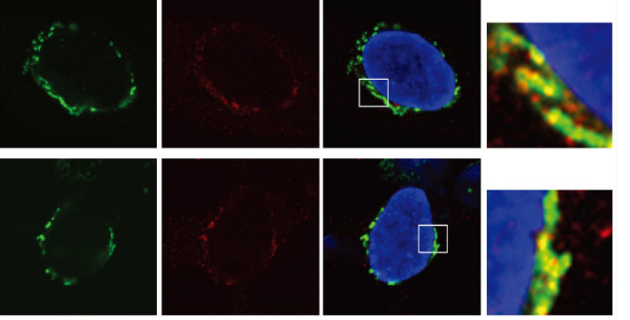

H279D
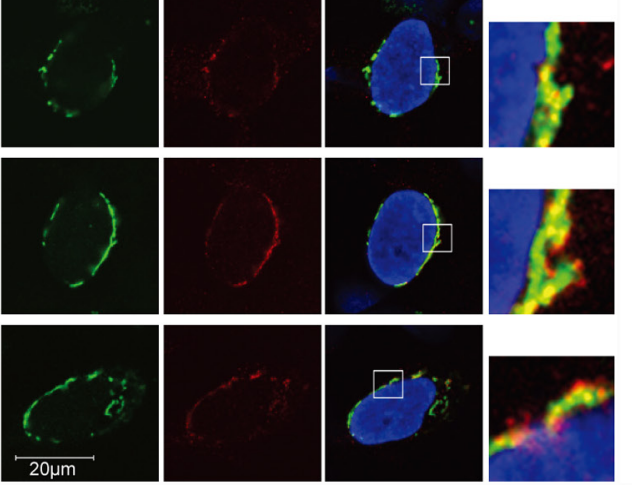

Tubulin

H415A
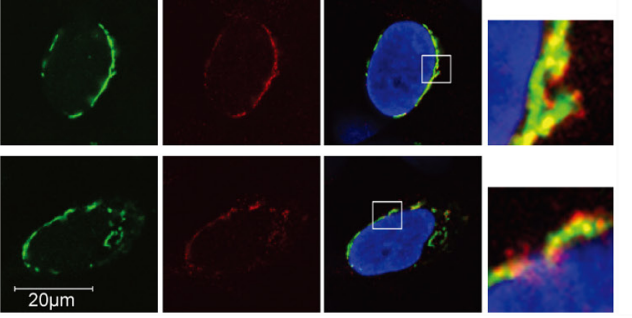

Fig. 2. Expression of wild-type and inactive mutants of GALNT3 in BEAS-2B cells. (A) The susceptibility of BEAS-2B cells for influenza A virus infection. The cells were infected with PR8 $(\mathrm{MOI}=3.0)$ and fixed at $12 \mathrm{hr}$ postinfection. Infection was detected by IFA using anti-NP antibody. Green signals indicate the virusinfected cells. (B) Western blot analysis of BEAS-2B cells stably or transiently expressing inactive mutants of GALNT3. Vector indicates the control cells transfected with empty plasmid. (C) Immunofluorescence analysis of GALNT3 on stably expressing BEAS-2B cells. The cell clones were stained with anti-GALNT3 polyclonal (green) and anti-Giantin (Golgi marker; Abcam) monoclonal (red) antibodies. Magnifications of boxed areas in merge panels are shown on the right. Blue indicates 4',6'-diamidino2-phenylindole (DAPI) staining of nuclei.
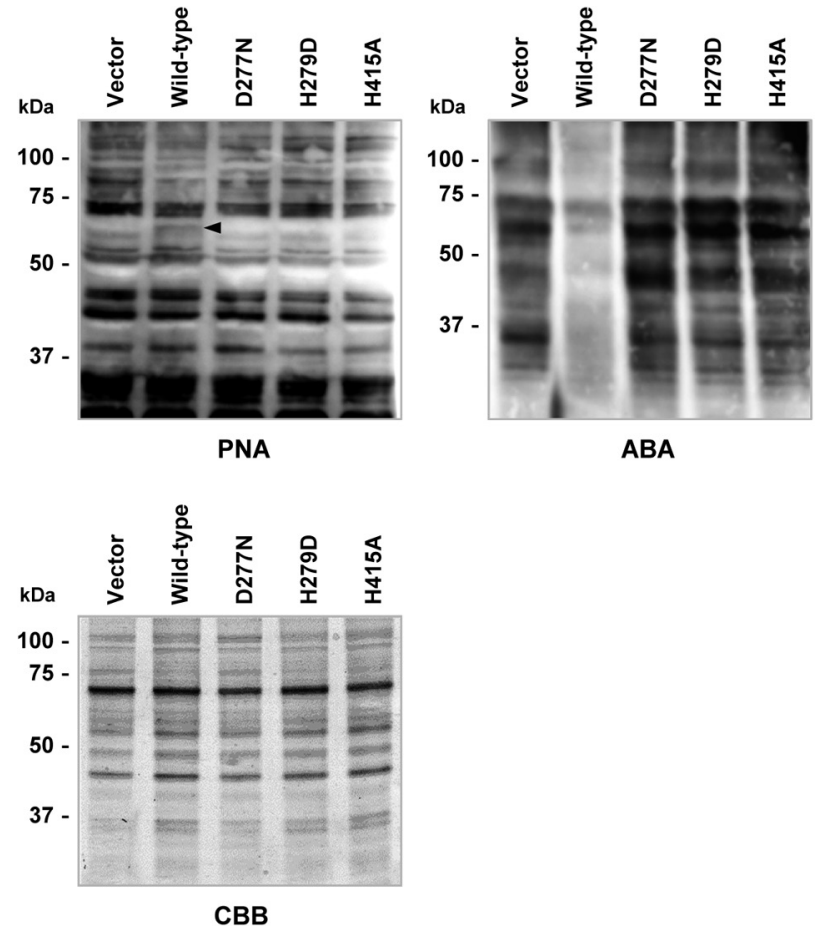

Fig. 3. Lectin blot analysis of stable cell clones expressing inactive mutants of GALNT3. Proteins from each cell lysate of wild-type and inactive mutant-expressing cell clones were subjected to $12 \%$ SDS-PAGE and lectin blot analysis. Lectin blotting was performed using PNA and ABA. Arrow indicates extra bands detected in the BEAS-2B cell line expressing wild-type GALNT3. CBB staining was also carried out on the same samples. After $1 \mathrm{hr}$ of staining with $\mathrm{CBB}$, the gel was destained in distilled water overnight to visualize protein bands.

Carbo-Free Blocking Solution for $1 \mathrm{hr}$ at room temperature. Because both PNA and ABA recognize the core of O-linked carbohydrate structures, particularly in Gal $\beta 1-3$ GalNAc units $[10,18]$, the enzyme activity of the GALNT3 could be detected by blotting using these lectins. After incubation with lectin, the membrane was washed 4 times with $0.05 \%$ Tween 20 in PBS and then incubated with streptavidin horseradish peroxidase conjugate for $1 \mathrm{hr}$ at room temperature. After washing, the blots were visualized using an enhanced chemiluminescence (ECL) capture system with Pierce Western Blotting Substrate Plus (Thermo Scientific, Rockford, IL, U.S.A.). As shown in Fig. 3, by using PNA, the cells expressing the wild-type GALNT3 seem to induce some extra bands, especially at $70 \mathrm{kDa}$ (arrowhead), when compared with the cells expressing the mutant constructs (Fig. 3). In addition, the blotting pattern of ABA seemed to be different in the high molecular weight region in the cells expressing wild-type GALNT3 from the mutant constructs. Although the blotting pattern of the wild-type clone by ABA appeared to be relatively weak compared with those of other clones, it might be due to the increase in nonspecific protein binding in the cells expressing vector and mutant plasmids. We repeated these experiments at least three times 
and always obtained the same results. Therefore, this result demonstrated that the mutants of GALNT3 generated here likely express inactive forms of GALNT3.

In this study, we generated human bronchial epithelial BEAS-2B cell lines stably expressing inactive mutants of GALNT3. A previous study revealed that anticipated glycosylation by different GALNT isoforms may negatively regulate the enzyme activities of subsequent GALNTs [5], suggesting that the stable expression of inactive mutants of GALNT3 may play a role as dominant negative isoforms in cells. Since the inactive GALNT3 generated here were expressed normally and showed the same intracellular localization as the wild-type protein, they may negatively control the activity of endogenous GALNT3. We are currently investigating this possibility using the cell lines generated in this study. Our data may provide valuable information for the creation of GALNT3-dominant negative mutants and/or mutations for enhancing GALNT3 activity. The cell lines expressing inactive mutants of GALNT3 would be useful tools in furthering our understanding the role of mucins in respiratory virus infection, as well as the host defense mechanism.

ACKNOWLEDGMENT. This study was supported in part by the Funding Program for Next Generation World-Leading Researchers of the Japan Society for the Promotion of Science (JSPS) and a grant from the Sumitomo Foundation.

\section{REFERENCES}

1. Brockhausen, I., Dowler, T. and Paulsen, H. 2009. Site directed processing: role of amino acid sequences and glycosylation of acceptor glycopeptides in the assembly of extended mucin type O-glycan core 2. Biochim. Biophys. Acta 1790: 1244-1257. [Medline] [CrossRef]

2. Excoffon, K. J., Gansemer, N. D., Mobily, M. E., Karp, P. H., Parekh, K. R. and Zabner, J. 2010. Isoform-specific regulation and localization of the coxsackie and adenovirus receptor in human airway epithelia. PLoS ONE 5: e9909. [Medline] [CrossRef]

3. Gu, C., Oyama, T., Osaki, T., Li, J., Takenoyama, M., Izumi, H., Sugio, K., Kohno, K. and Yasumoto, K. 2004. Low expression of polypeptide GalNAc $N$-acetylgalactosaminyl transferase-3 in lung adenocarcinoma: impact on poor prognosis and early recurrence. Br. J. Cancer 90: 436-442. [Medline] [CrossRef]

4. Hagen, F. K., Hazes, B., Raffo, R. and Tabak, L. A. 1999. Structure-function analysis of the UDP- $N$-acetyl-Dgalactosamine:polypeptide $N$-acetylgalactosaminyltransferase. Essential residues lie in a predicted active site cleft resembling a lactose repressor fold. J. Biol. Chem. 274: 6797-6803. [Medline] [CrossRef]

5. Hanisch, F. G., Müller, S., Hassan, H., Clausen, H., Zachara, N., Gooley, A. A., Paulsen, H., Alving, K. and Peter-Katalinic, J. 1999. Dynamic epigenetic regulation of initial $O$-glycosylation by UDP- $N$-acetylgalactosamine:peptide $N$-acetylgalactosaminyltransferases. Site-specific glycosylation of MUC1 repeat peptide influences the substrate qualities at adjacent or distant Ser/Thr positions. J. Biol. Chem. 274: 9946-9954. [Medline]
[CrossRef]

6. Ioannidis, I., McNally, B., Willette, M., Peeples, M. E., Chaussabel, D., Durbin, J. E., Ramilo, O., Mejias, A. and Flaño, E. 2012. Plasticity and virus specificity of the airway epithelial cell immune response during respiratory viral infection. J. Virol. 86: 5422-5436. [Medline] [CrossRef]

7. Kato, K., Jeanneau, C., Tarp, M. A., Benet-Pagès, A., LorenzDepiereux, B., Bennett, E. P., Mandel, U., Strom, T. M. and Clausen, H. 2006. Polypeptide GalNAc-transferase T3 and familial tumoral calcinosis. Secretion of fibroblast growth factor 23 requires O-glycosylation. J. Biol. Chem. 281: 18370-18377. [Medline] [CrossRef]

8. Matrosovich, M. N., Matrosovich, T. Y., Gray, T., Roberts, N. A. and Klenk, H. D. 2004. Neuraminidase is important for the initiation of influenza virus infection in human airway epithelium. J. Virol. 78: 12665-12667. [Medline] [CrossRef]

9. Miller, A. L., Strieter, R. M., Gruber, A. D., Ho, S. B. and Lukacs, N. W. 2003. CXCR2 regulates respiratory syncytial virusinduced airway hyperreactivity and mucus overproduction. $J$. Immunol. 170: 3348-3356. [Medline]

10. Nakamura-Tsuruta, S., Kominami, J., Kuno, A. and Hirabayashi, J. 2006. Evidence that Agaricus bisporus agglutinin (ABA) has dual sugar-binding specificity. Biochem. Biophys. Res. Commun. 347: 215-220. [Medline] [CrossRef]

11. Röttger, S., White, J., Wandall, H. H., Olivo, J. C., Stark, A., Bennett, E. P., Whitehouse, C., Berger, E. G., Clausen, H. and Nilsson, T. 1998. Localization of three human polypeptide GalNAc-transferases in HeLa cells suggests initiation of $O$-linked glycosylation throughout the Golgi apparatus. J. Cell Sci. 111: 45-60. [Medline]

12. Rudd, B. D., Smit, J. J., Flavell, R. A., Alexopoulou, L., Schaller, M. A., Gruber, A., Berlin, A. A. and Lukacs, N. W. 2006. Deletion of TLR3 alters the pulmonary immune environment and mucus production during respiratory syncytial virus infection. $J$. Immunol. 176: 1937-1942. [Medline]

13. Sabbah, A. and Bose, S. 2009. Retinoic acid inducible gene I activates innate antiviral response against human parainfluenza virus type 3. Virol. J. 6: 200. [Medline] [CrossRef]

14. Sanders, C. J., Doherty, P. C. and Thomas, P. G. 2011. Respiratory epithelial cells in innate immunity to influenza virus infection. Cell Tissue Res. 343: 13-21. [Medline] [CrossRef]

15. Tarp, M. A. and Clausen, H. 2008. Mucin-type $O$-glycosylation and its potential use in drug and vaccine development. Biochim. Biophys. Acta 1780: 546-563. [Medline] [CrossRef]

16. Wandall, H. H., Hassan, H., Mirgorodskaya, E., Kristensen, A. K., Roepstorff, P., Bennett, E. P., Nielsen, P. A., Hollingsworth, M. A., Burchell, J., Taylor-Papadimitriou, J. and Clausen, H. 1997. Substrate specificities of three members of the human UDP- $N$-acetyl- $\alpha$-D-galactosamine:polypeptide $N$-acetylgalactosaminyltransferase family, GalNAc-T1, -T2, and -T3. J. Biol. Chem. 272: 23503-23514. [Medline] [CrossRef]

17. Wragg, S., Hagen, F. K. and Tabak, L. A. 1997. Identification of essential histidine residues in UDP- $N$-acetyl-Dgalactosamine:polypeptide $\mathrm{N}$-acetylgalactosaminyltransferaseT1. Biochem. J. 328: 193-197. [Medline]

18. Wu, W., Punt, J. A., Granger, L., Sharrow, S. O. and Kearse, K. P. 1997. Developmentally regulated expression of peanut agglutinin (PNA)-specific glycans on murine thymocytes. Glycobiology 7: 349-356. [Medline] [CrossRef] 